\title{
Chronic diseases like asthma and COPD: do they truly exist?
}

\author{
Peter J. Sterk \\ Affiliation: Dept of Respiratory Medicine, Academic Medical Centre, University of Amsterdam, Amsterdam, \\ The Netherlands. \\ Correspondence: Peter J. Sterk, Dept of Respiratory Medicine, F5-259, Academic Medical Centre, University

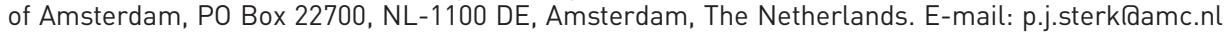

@ERSpublications

Historical diagnoses of asthma and COPD should be replaced with bioclinical phenotyping http://ow.ly/VulR0

We have all been taught what asthma is and currently, the worldwide Global Initiative for Asthma (GINA) strategy defines the disease in great detail [1]. Similarly, chronic obstructive pulmonary disease (COPD) has been delineated carefully by the Global Initiative for Chronic Obstructive Lung Disease (GOLD), which provides concrete recommendations for its diagnosis based on today's standards [2]. These two efforts have probably done more for the benefit of patients with chronic airway diseases around the world than any top-level research programme in the field: chapeau! The solid recommendations by GINA and GOLD are based on knowledge accumulated since, let us say, the mid-20th century regarding the background, assessment and management of these established diseases. Hence, would there be any reason for shaking up asthma and COPD?

\section{Disease?}

What makes a condition an established disease? Even though in medical school, we may have taken it for granted that diseases like asthma and COPD actually exist, what did it take to get these diseases into our textbooks? For asthma, this was based on centuries of clinical recognition [3], whereas the history of COPD starts around 1945, obviously with longer roots related to chronic bronchitis and emphysema. Regardless of the exact terminologies, it is obvious to everyone working in day-to-day care that clinical presentations of patients match particular categories, such as those labelled as asthma or COPD. When a patient is exhibiting episodic chest symptoms together with variable airway obstruction, we confidently categorise this as an asthma case. The question, then, is whether all cases presenting this way represent a true disease.

Disease can be defined as an "abnormal" condition, reflected by a disorder in structure or function, affecting part or all of an organism [4]. First, what can be regarded as abnormal can be disputed. The complex structural and functional networks of a living being are not stable and are continuously fluctuating between particular degrees of order and chaos [5], thereby driving an organism into various states that can either be in concert with the demands of wellbeing and survival on this planet (adapted, health) or not (not adapted, disease). Hence, disease is a qualitative label for a status of an organism obtained by physiological variation, in which the distinction with pathophysiology is not unambiguous and dependent on adaptation to its environment. In particular, this holds for chronic diseases.

Second, the presence of a particular disease is derived from carefully observing an individual. Traditionally, this has entirely been driven by clinical symptoms and signs. Pattern recognition of those symptoms and signs has subsequently led to the delineation of particular commonly encountered entities (diseases) and their diagnostic criteria. Thereby, the diagnosis of asthma is merely based on human pattern recognition based on the modes of observation that were applicable during the 18th and 19th century. Does such pattern recognition of historically available features adequately capture comprehensive disorders in the structure and function of an organism?

Received: Nov 192015 | Accepted: Nov 192015

Conflict of interest: None declared.

Copyright @ERS 2016 


\section{Phenotyping}

In this issue of the European Respiratory Journal, Agusti et al. [6] make up the 2016 balance for asthma and COPD, by promoting a taxonomy based on treatable traits. They rightly argue that today's modes of observing the patient are endlessly richer than those available when these historical diagnoses were framed. Similar to all organisms, human beings represent a self-organising biological system that is essentially dynamic and nonlinear, representing the essence of life [7]. Therefore, whenever we wish to define a "normal" or "abnormal" state of a person in front of us, we must include the underlying complex biology. Then, observing the patient not only includes symptoms and signs, but also other phenomena such as cellular, molecular and morphometric parameters. For chronic airway diseases like asthma and COPD, AgUSTI et al. [6] not only propose pulmonary function and airway hyperresponsiveness to be included for capturing the traits, but also computed tomography, and cellular and molecular markers in blood, sputum and exhaled air, together with sensitive microbial analysis. These measures are meant to capture phenotypes of chronic airway disease that can be linked to evidence-based therapeutic choices.

This concept of adequately phenotyping patients with airway disease was originally launched by DE VRIES et al. [8] in 1968, who at that time promoted the usage of various inhalation challenge tests to obtain an individual phenotype of a patient with chronic airway disease. Now, we can sample biology and (ultra) structure, which eventually provides phenotypes that more precisely represent the status of the organism. This is not only suitable for more adequately selecting patients for existing or newly available therapies (treatable traits), but also for adequately describing comorbidities and for as-yet nontreatable traits that require the development of novel treatment modalities. These novel phenotypes may even lead to renewed discovery of true diseases when all the causal, pathogenetic pathways have been unravelled. In such cases, we are dealing with so-called endotypes [9], which unfortunately have scarcely, if ever, been established amongst airway diseases. Hence, establishing the bio-clinical phenotype of a patient with chronic airway disease will be far more informative than just using the historical diagnosis of asthma or COPD [6].

\section{Farewell asthma, COPD and asthma-COPD overlap syndrome}

What will be the implication of advocating such a new level of preciseness in chronic airway disease? It can be envisaged that AGUSTI et al. [6] are merely marking the beginning of a new era. Today, biology cannot only be captured by pre-selected molecular measurements, but also by composite platforms that are mapping the entire mixture of molecular samples from RNA, protein or metabolic networks [10]. As we speak, this is being applied in respiratory research [11], which has opened several new windows, in parallel, to more comprehensive biological phenotyping of chronic airway diseases based on quantitative probability analysis by pattern recognition algorithms. There is little doubt that precision phenotyping and personalised respiratory medicine will increasingly rely on such "systems" approaches, for which clear guidance has been provided, recently, in terms of: 1) the strategies [12], 2) the mathematical algorithms [13] and stringent quality criteria [14].

As it appears, at present, we are more or less in a transitional phase between using traditional diagnoses based on historical observations on the one hand and newly discovered bio-clinical phenotypes based on "systems approaches" on the other. For example, recent American Thoracic Society/European Respiratory Society recommendations on research priorities still use the historical diagnosis of COPD as leading principle [15]. Even U-BIOPRED (www.ubiopred.eu), a pan-European systems medicine project, is using patients who meet the criteria for a diagnosis of (severe) asthma as starting point $[16,17]$ rather than abandoning such historical disease description. Perhaps most illustrative for the traditional view on airways diseases is the recent clinical label of the ACOS (asthma-COPD overlap syndrome) [18, 19]. Is not this archetypic traditional thinking? Cannot this be considered the ultimate attempt to rescue historical concepts by putting a new clinical label on conditions where the biology clearly shows emerging phenotypes that do not follow the incomplete, traditional classifications or their inevitable clinical overlap?

\section{Let us be brave}

It is somewhat amusing that, ultimately, Agusti et al. [6] do not push their idea all the way. Rather than abandoning the historical diagnoses of asthma and COPD, they recommend using their treatable traits: "complement and refine' the diagnostic labels of asthma and COPD (as used in the respective GINA...and GOLD...reports)" [6]. The reasoning is that the evidence for effectively using the phenotyping approach is still incomplete. However, it is about time to turn this argument around. What is the evidence that the historical diagnoses add useful information to detailed phenotyping of the patient based on multiple clinical and biological characteristics? There is no such evidence. This is not unexpected, since all the information required to decide whether a patient fits the historical diagnosis of asthma or COPD according to GINA or GOLD criteria is fully included in the more comprehensive phenotypic information, as listed by AGUsTi et al. [6] in table 1 of their article. Hence, providing a diagnostic label based on a historical subset of phenotypic characteristics will, by definition, not provide complementary information. 
Therefore, let us be brave. Ever since De VRIES et al. [8] in 1968, it has been tempting to guide patient management by phenotypic characteristics rather than diagnostic labels. Now, almost 50 years later, with all our biological knowledge and new targets for therapy, we are still not prepared to bite the bullet. Historical diagnoses are impeding modern medicine. Let us follow the botanists, who have gained a wealth of new knowledge when abandoning their historical taxonomy and incorporating molecular biological data. Of course, one may question whether it will ever be possible to capture biology in health and disease at its full complexity. Even when using all the omics and imaging in the world, living organisms and their biology may escape us because they are not static and are intrinsically dynamic, based on homeokinesis rather than homeostasis [5, 7]. We cannot keep on measuring all day, can we? Eventually, we may go the same way as the physicists, who are accepting absolute and definitive limits of capturing reality, and frankly, that is not a bad act to follow.

\section{References}

1 Reddel HK, Bateman ED, Becker A, et al. A summary of the new GINA strategy: a roadmap to asthma control. Eur Respir J 2015; 46: 622-639.

2 Vestbo J, Hurd SS, Agusti AG, et al. Global strategy for the diagnosis, management, and prevention of chronic obstructive pulmonary disease: GOLD executive summary. Am J Respir Crit Care Med 2013; 187: 347-365.

3 Brewis RAL ed. Classic Papers in Asthma. Volume 1. The Evolution of Understanding. London, Science Press, 1990. Dorland's Illustrated Medical Dictionary. 31st Edn. Philadelphia, Elsevier Saunders, 2007.

Macklem PT, Seely A. Towards a definition of life. Perspect Biol Med 2010; 53: 330-340.

Agusti A, Bel EH, Thomas M, et al. Treatable traits: toward precision medicine of chronic airway disease. Eur Respir J 2016; 47: 410-419.

7 Periyasamy S, Gray A, Kille P. The bottom-up approach to defining life: deciphering the functional organization of biological cells via multi-objective representation of biological complexity from molecules to cells. Front Physiol 2013; 4: 369 .

8 De Vries K, Booy-Noord H, van der Lende R, et al. Reactivity of the bronchial tree to different stimuli. Bronches 1968; 18: 439-452.

9 Anderson GP. Endotyping asthma: new insights into key pathogenetic mechanisms in a complex, heterogeneous disease. Lancet 2008; 372: 1107-1119.

10 Chen R, Mias GI, Li-Pook-Than J, et al. Personal omics profiling reveals dynamic molecular and medical phenotypes. Cell 2012; 148: 1293-1307.

11 Wheelock CE, Goss VM, Balgoma D, et al. Application of 'omics technologies to biomarker discovery in inflammatory lung diseases. Eur Respir J 2013; 42: 802-825.

12 Agusti A, Anto JM, Auffray C, et al. Personalized respiratory medicine: exploring the horizon, addressing the issues. Am J Respir Crit Care Med 2015; 191: 391-401.

13 Sung J, Wang Y, Chandrasekaran S, et al. Molecular signatures from omics data: from chaos to consensus. Biotechnol J 2012; 7: 946-957.

14 McShane LM, Cavenagh MM, Lively TG, et al. Criteria for the use of omics-based predictors in clinical trials: explanation and elaboration. BMC Med 2013; 11: 220-200.

15 Celli BR, Decramer M, Wedzicha JA, et al. An official American Thoracic Society/European Respiratory Society statement: research questions in COPD. Eur Respir J 2015; 24: 159-172.

16 Fleming L, Murray C, Bansai AT, et al. The burden of severe asthma in childhood and adolescence: results from the paediatric U-BIOPRED cohorts. Eur Respir J 2015; 46: 1322-1333.

17 Shaw DE, Sousa AR, Fowler SJ, et al. Clinical and inflammatory characteristics of the European U-BIOPRED adult severe asthma cohort. Eur Respir J 2015; 46: 1308-1321.

18 Postma DS, Rabe KF. The asthma-COPD overlap syndrome. N Engl J Med 2015; 373: 1241-1249.

19 Bateman ED, Reddel HK, van Zyl-Smit RN, et al. The asthma-COPD overlap syndrome: towards a revised taxonomy of chronic airways diseases? Lancet Respir Med 2015; 3: 719-728. 